\title{
The cysteine-cluster motif of c-Yes, Lyn and FAK as a suppressive module for the kinases
}

\author{
MOHAMMAD AMINUR RAHMAN ${ }^{1 *}$, TAKESHI SENGA $^{1 *}$, MYAT LIN OO $^{1 *}$, \\ HITOKI HASEGAWA ${ }^{1}$, MD. HELAL UDDIN BISWAS ${ }^{1}$, NAING NAING MON ${ }^{1}$, PENGYU HUANG ${ }^{1}$, \\ SATOKO ITO $^{1}$, TADASHI YAMAMOTO ${ }^{2}$ and MICHINARI HAMAGUCHI $^{1}$ \\ ${ }^{1}$ Division of Cancer Biology, Nagoya University School of Medicine, Nagoya 466-8550; \\ ${ }^{2}$ Department of Oncology, Institute of Medical Science, Tokyo University, \\ 4-6-1 Shirokanedai, Minato-ku, Tokyo 108-8639, Japan
}

Received September 5, 2007; Accepted November 12, 2007

\begin{abstract}
The Src family of non-receptor protein tyrosine kinases plays a critical role in the progression of human cancers so that the development of its specific inhibitors is important as a therapeutic tool. We previously reported that cysteine residues in the cysteine-cluster (CC) motif of v-Src were critical for the kinase inactivation by the SH-alkylating agents such as $\mathrm{N}$-(9-acridinyl) maleimide (NAM), whereas other cysteine residues were dispensable. We found similar CC-motifs in other Src-family kinases and a non-Src-family kinase, FAK. In this study, we explored the function of the CC-motif in Yes, Lyn and FAK. While Src has four cysteines in the CC-motif, c-Yes and Lyn have three and two of the four cysteines, respectively. Two conserved cysteines of the Src family kinases, corresponding to Cys487 and Cys498 of Src, were essential for the resistance to the inactivation of the kinase activity by NAM, whereas the first cysteine of c-Yes, which is absent in Lyn, was less important. FAK has similar CC-motifs with two cysteines and both cysteines were again essential for the resistance to the inactivation of the kinase activity by NAM. Taken together, modification of cysteine residues of the CC-motif causes a repressor effect on the catalytic activity of the Src family kinases and FAK.
\end{abstract}

\section{Introduction}

Since the discovery of the $\mathrm{v}$-Src kinase (1), many protein tyrosine kinases have been identified. Overexpression as well

Correspondence to: Dr Michinari Hamaguchi, Division of Cancer Biology, Nagoya University School of Medicine, 65 Tsurumai-cho, Showa-ku, Nagoya 466-8550, Japan

E-mail: mhamagu@med.nagoya-u.ac.jp

${ }^{*}$ Contributed equally

Key words: c-Yes, Lyn, FAK, SH-alkylating agent N-(9-acridinyl) maleimide, kinase inactivation, cysteine as the constitutive activation of tyrosine kinases are associated with many forms of human cancer. It appears that $>80 \%$ of the oncogenes and proto-oncogenes involved in human cancers encode tyrosine kinase $(2,3)$ so that the development of their specific inhibitors is an important mission for the therapeutic progress of human malignancies. All kinases, however, share the same substrate, adenosine 5'-triphosphate (ATP) and most, if not all, of kinase inhibitors target their conserved nucleotide binding pocket so that no inhibitor with a highly selective inhibitory effect on a single kinase has been found (4). Despite the wide variety in the kinase structure, all the inhibitors identified have some redundancy in their specificity.

Investigating an alternative way to control specific types of tyrosine kinases, we explored the cysteine residues of Src which is known to be critical for its kinase activity $(5,6)$. The viral oncogene product, v-Src and its cellular counterpart, $\mathrm{c}-\mathrm{Src}$, are members of a family of non-receptor protein tyrosine kinase called the Src-family kinase that shares structural similarity consisting of the $\mathrm{SH} 2, \mathrm{SH} 3$ and kinase domains (1). While the c-Src kinase is overexpressed and activated in a large number of human malignancies, it has been linked to the development of cancer and progression to distant metastases (7). Activity of the Src kinase is controlled by the intramolecular bindings of SH2 to phosphorylated Tyr527 and of SH3 to the linker region. These interactions appear to fold the c-Src molecule to be in an inactive state (8-10). Any events or mutations including a point mutation at Tyr527 that unfold the structure can convert c-Src to be active and highly oncogenic $(8,11)$. In addition to these domains, we identified a new regulatory motif, the cysteine-cluster $(\mathrm{CC})$ motif, at the end of the $\mathrm{C}$-terminal lobe of Src where four cysteine residues are clustered $(5,6)$. Of the 10 cysteine residues scattered over the $\mathrm{v}$-Src kinase, only those in the CC-motif were important for protein stability and cell transformation (6). Consistently, we found that the SH-alkylating agents could inactivate the Src kinase, though mutations of cysteine residues, especially Cys498, in the CC-motif rendered v-Src and $\mathrm{c}-\mathrm{Src}$ to be resistant to the inactivation of the kinase activity by the SH-alkylating agents (5). In contrast, other cysteines including those in the catalytic cleft of the kinase 

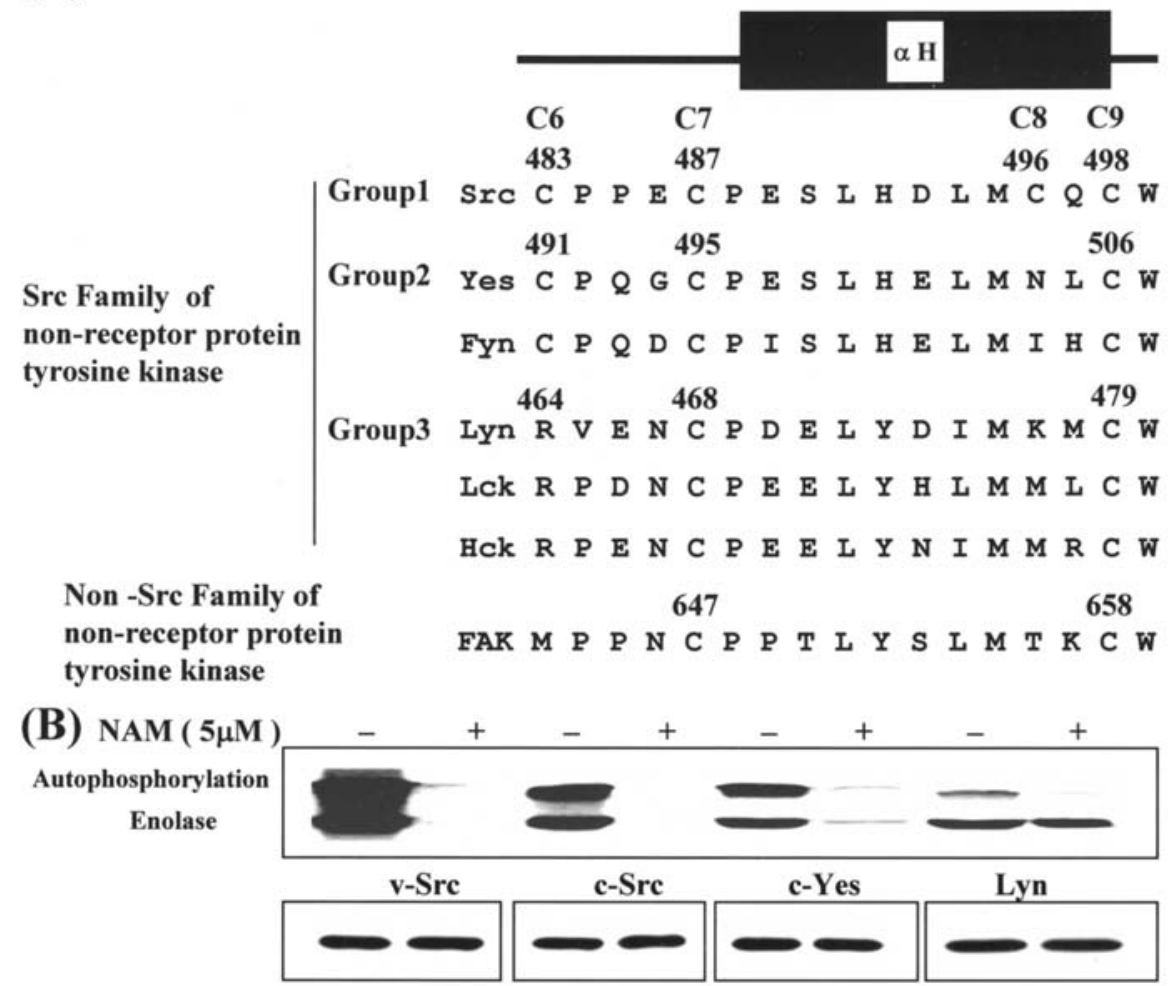

Figure 1. The primary sequences and secondary structural alignment of the CC-motif of the Src-family kinases and FAK and their response to the SH-alkylating agent, NAM. (A) Sequence text was constructed for the Src-family kinases and FAK, as previously described (5). Based on the amino acid-matching of the cysteine residues in the CC-motif that corresponds to the sequence of v-Src from 481Met to 499Trp, the Src-family kinases and FAK were classified into three groups. Secondary-protein structural alignment given above the sequences was based on a previous report (5). (B) Kinase genes ligated into pcDNA3 were trasfected into COS-7 cells and lysates were collected after $36 \mathrm{~h}$ of transfection. Kinase proteins were immunoprecipitated with their related antibodies, incubated in the presence or absence of $5 \mu \mathrm{M}$ NAM and subjected to an in vitro kinase assay at $30^{\circ} \mathrm{C}$ for 15 min with enolase (upper panel) as an exogenous substrate. Their relative amounts were normalized by immunoblotting with their specific antibodies to use the same amounts of kinase proteins for the study (lower panel).

domain and in the $\mathrm{SH} 2$ domain were dispensable for the inactivation by the SH-alkylating agents. Thus, modification of cysteine residues in the CC-motif of Src kinase appears to yield a conformational change that causes a suppressive effect on the catalytic activity of Src.

Among the Src-family kinases, the CC-motif is highly conserved, while all receptor-type tyrosine kinases and other nonreceptor-type tyrosine kinases such as Abl lack this motif. In addition to the Src-family kinases, we identified the same motif in another type of tyrosine kinase, FAK. It is, therefore, of interest whether modification of cysteine residues in the $\mathrm{CC}$-motifs of these kinases has a similar suppressive function on their kinase activities. Herein, we show evidence that cysteine residues in the $\mathrm{CC}$-motif are important for the inhibition of the Yes, Lyn and FAK by the SH-alkylating agent $\mathrm{N}$-(9-acridinyl) maleimide (NAM).

\section{Materials and methods}

Cell culture, antibodies and chemicals. COS-7 cells were cultured as previously described (5). Anti-Src monoclonal antibody, mAb 327, was kindly provided by Dr J.S. Brugge (Harvard Medical School) (12). Anti-Yes and anti-Lyn monoclonal antibodies were purchased from BD Transduction Laboratories and anti-FAK polyclonal antibody from Santa Cruz Biotechnology. NAM was from Wako Pure Chemical
Industries, Ltd. Casein, enolase and poly (Glu,Tyr) were purchased from Sigma.

Site-directed mutagenesis and transfection. Cysteine residues in c-Yes (13), Lyn (14) and FAK (15) were substituted to alanines by PCR as previously reported (16). Mutated $c$-yes, lyn and $F A K$ were ligated into the TOPO TA Cloning vector (pCR $\left.{ }^{\circledR} 2.1-\mathrm{TOPO}\right)$ and sequenced. Mutated $c$-yes, lyn and Fak were cut by a suitable restriction enzyme, collected and ligated into a pcDNA3 vector and reconfirmed the insertion by sequencing. Each mutated gene ( $5 \mu \mathrm{g}$ of each) in pcDNA3 was transiently transfected into COS-7 cells using DEAEdextran/chloroquine solution.

In vitro kinase assay and immunoblotting. An in vitro kinase assay was performed as previously described $(17,18)$. In brief, cells were lysed with RIPA buffer $(10 \mathrm{mM}$ Tris- $\mathrm{HCl} \mathrm{pH} 7.4$, $150 \mathrm{mM} \mathrm{NaCl}, 5 \mathrm{mM}$ EDTA, $1 \%$ Triton $\mathrm{X}-100$, $1 \%$ sodium deoxycholate, $0.1 \%$ SDS, $0.1 \mathrm{mM}$ molybdic acid and $0.5 \mathrm{mM}$ sodium orthovanadate and $0.1 \mathrm{mM}$ phenylmethylsulfonyl fuoride and proteinase inhibitor cocktail from Sigma). The lysate was clarified by centrifugation and immunoprecipitated with antibodies. The immunoprecipitates were subjected to either immunoblotting or an in vitro kinase assay.

For the treatment with NAM, immunoprecipitates were washed with kinase buffer $(10 \mathrm{mM}$ Tris $-\mathrm{HCl} \mathrm{pH} 7.4,5 \mathrm{mM}$ 


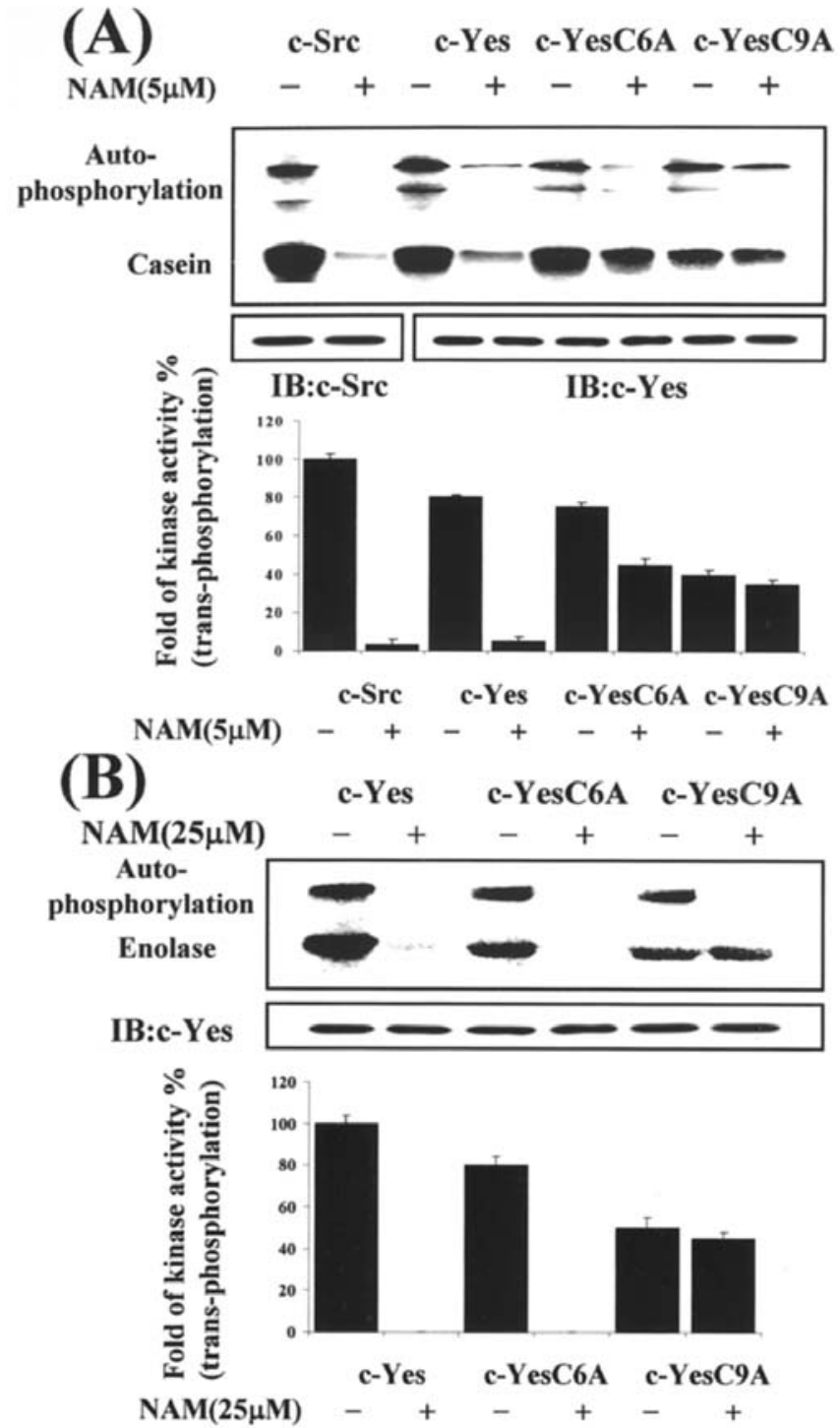

Figure 2. Effect of SH-alkylating agent, NAM, on the kinase activities of wild-type c-Src (group 1), wild-type c-Yes (group 2) and c-Yes mutants, C6AYes and C9AYes. (A) Cell lysates (500 $\mu \mathrm{g}$ of total protein) from transiently transfected COS-7 cells with wild-type or mutant kinase genes were immunoprecipitated with anti-Src (mAb 327) or anti-Yes monoclonal antibody. Immunoprecipitates were incubated in the presence or absence of $5 \mu \mathrm{M}$ of NAM and a kinase assay was performed with casein as an exogenous substrate (upper panel). Immunoprecipitates were probed with indicated antibodies to confirm an equal amount of loading (middle panel). Relative activities of an in vitro kinase assay were measured by the substrate transphosphorylation with BAS 2000 image analyzer (Fuji). Results were obtained from three independent assays (lower panel). (B) A higher dose of NAM $(25 \mu \mathrm{M})$ was used as above for the treatment of immunoprecipitates and enolase was used as an alternative exogenous substrate.

$\mathrm{MgCl}_{2}$ for the Src family and $50 \mathrm{mM}$ Hepes $\mathrm{pH} 7.4,10 \mathrm{mM}$ $\mathrm{MnCl}_{2}$ for FAK ), suspended in the same buffer and incubated with or without the indicated doses of NAM for $5 \mathrm{~min}$ at $30^{\circ} \mathrm{C}$. After the reaction, immunoprecipitates were washed twice and incubated in kinase buffer with radiolabeled $\left[\gamma^{32} \mathrm{P}\right]$ ATP $(370 \mathrm{kBq})(\mathrm{NEN}$, Wilmington, DE, USA) and $1.5 \mu \mathrm{g}$ of acid-treated enolase, $1 \mu \mathrm{g}$ of pure casein or $1 \mu \mathrm{g}$ of poly (Glu-Tyr) as an exogenous substrate. After incubation of 15 min for the Src family and $2 \mathrm{~h}$ for $\mathrm{FAK}$ at $30^{\circ} \mathrm{C}$, reaction was stopped by the addition of an SDS sample buffer. The immunoprecipitates were then subjected to $10 \%$ SDS- polyacrylamide gel electrophoresis. Gels were dried and exposed to an IP plate and analysed by the BAS 2000 system. Immunoblotting was performed as described (19).

\section{Results}

CC-motif is conserved among Src family kinases. We previously reported that the CC-motif of Src has a suppressive function on the catalytic activity of the Src kinase by use of the $\mathrm{SH}$-alkylating agents and mutant Src proteins (5). In contrast to the cysteines in the CC-motif, other cysteine residues, including those in the catalytic cleft of the kinase domain and in the $\mathrm{SH} 2$ domain, were dispensable for the kinase inactivation by the SH-alkylating agents. We found that multiple mutations, though not a single point mutation, of the cysteine residues of the CC-motif conferred the resistance to the kinase inactivation by the $\mathrm{SH}$-alkylating agents. During the course of the study, we found that the CC-motif is well conserved among other members of the Src-family kinases. Based on the numbers and positions of cysteine residues in the CC-motif, the Src-family kinases can be classified into 3 groups (Fig. 1A). $\mathrm{v}-\mathrm{Src}$ and its cellular counterpart c-Src have a complete set of 4 cysteines (Cys483, Cys487, Cys496 and Cys498) in the CC-motif that starts from 481Met and ends at 499Trp of the v-Src sequence. In contrast, c-Yes, Fyn, Yrk and Blk (mouse) have 3 cysteine residues except for the one corresponding to Cys496 of Src. Lyn, Hck, Lck, Blk (human) and Frk have 2 cysteines corresponding to Cys487 and Cys498 of Src. In addition to these Src-family kinases, FAK, which does not belong to the Src-family kinase, also has the CC-motif similar to Lyn.

Effect of SH-alkylating agent, NAM, on the kinase activities of Src family kinases. To explore the function of the CC-motif in the second and third groups of these kinases, we chose c-Yes and Lyn to compare with v-Src and c-Src. These kinase genes were ligated into pcDNA3 and transiently expressed in COS-7 cells. Each kinase protein was immunoprecipitated with its specific antibody. Their relative amounts were normalized by immunoblotting with their specific antibodies to use the same amounts of kinase proteins for the study (Fig. 1B, lower panel). Immunoprecipitated c-Yes, Lyn, v-Src and c-Src were incubated with or without $5 \mu \mathrm{M}$ NAM at $30^{\circ} \mathrm{C}$ for $5 \mathrm{~min}$, washed with kinase buffer and subsequently assayed for their kinase activities with enolase as an exogenous substrate (Fig. 1B, upper panel). In accordance with our previous report, the kinase activities of v-Src and c-Src were almost completely inactivated by the treatment with NAM. c-Yes was also highly sensitive to the level similar to those of v-Src and c-Src. In contrast, the kinase activity of Lyn, especially the phosphorylation of the exogenous substrate, was substantially resistant to the treatment with $5 \mu \mathrm{M}$ NAM. These results are consistent with our previous observation that, at least, double mutations of cysteine residues of the CC-motif were required for Src kinase to exhibit resistance to the inactivation by NAM (5). As shown in Fig. 1A, Lyn lacks two cysteine residues which correspond to Cys483 and Cys496 of Src.

C9AYes mutant shows resistance to kinase inactivation by $N A M$. To obtain more clues, we then constructed two types of 

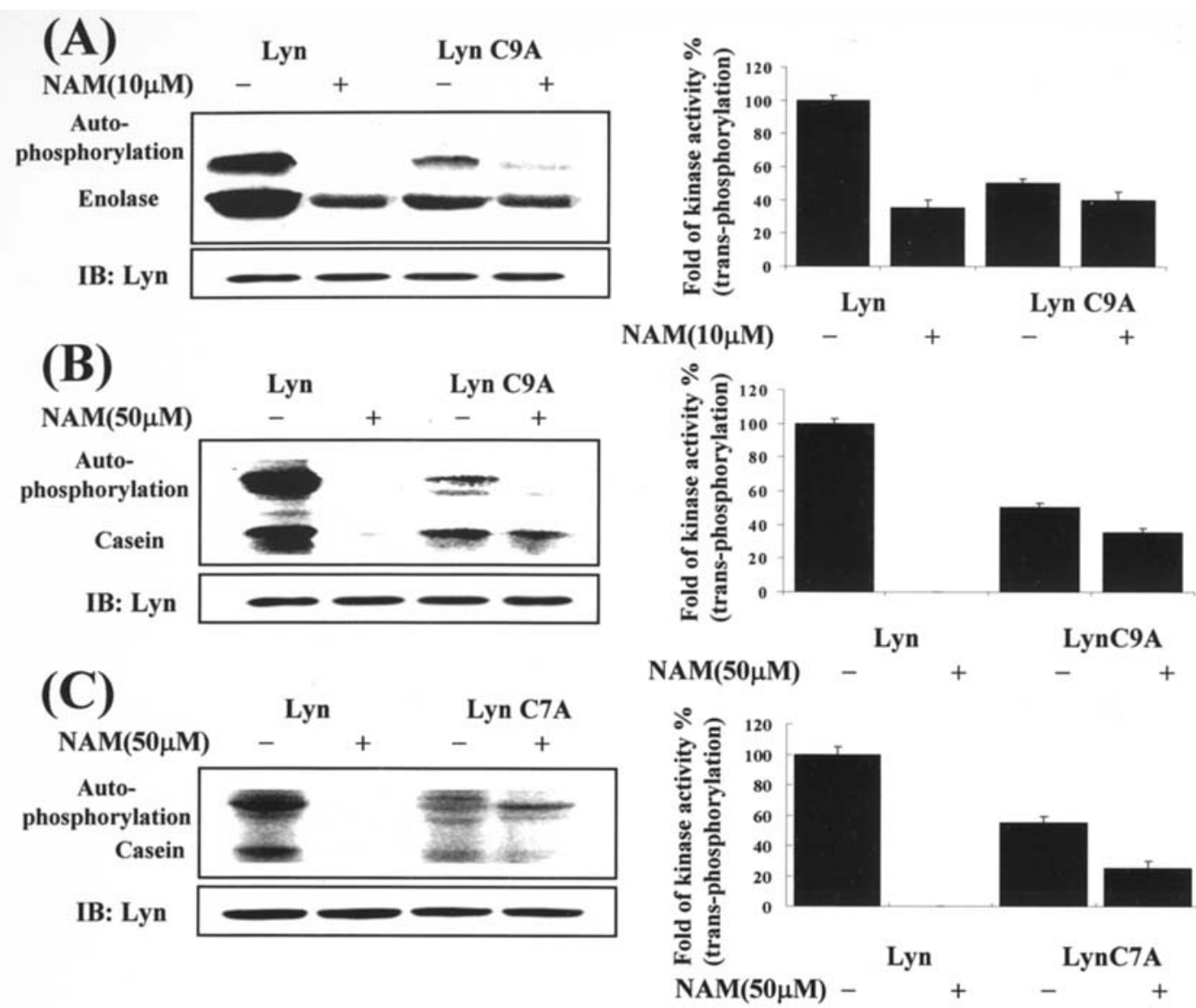

Figure 3. Effect of the SH-alkylating agent, NAM, on the in vitro kinase activities of wild-type Lyn (group 3), LynC7A and LynC9A. (A) Lyn and its mutant, LynC9A, were treated with $10 \mu \mathrm{M}$ NAM and a kinase assay was performed (left, upper panel) with enolase as an exogenous substrate as described in Fig. 2. Immunoprecipitates were probed with anti-Lyn to confirm an equal amount of loading (left, lower panel). Relative activities of the in vitro kinase assay were measured by the trans-phosphorylation of enolase (right panel). (B and C) In vitro kinase assay of Lyn, LynC9A and LynC7A mutants in the presence or absence of high-dose NAM $(50 \mu \mathrm{M})$ using casein as an exogenous substrate was performed as above. All figures are representative of three independent experiments.

Yes mutants, C6AYes and C9AYes. In C6AYes and C9AYes, Cys491 and Cys506 of Yes were replaced with alanine residues by site-directed mutagenesis (6), respectively. As shown in Fig. 2A, both mutants had reduced kinase activities (trans-phosphorylation to exogenous substrates) compared with that of wild-type Yes. A reduction of kinase activity is, however, more prominent in C9AYes as compared with that of C6AYes. Kinase activities of C9A and C6A of Yes mutants were $\sim 50$ and $80 \%$ of wild-type c-Yes, respectively. These results are consistent with our previous observation (5) that C9A v-Src, whose Cys498 was replaced with alanine, displayed relatively low kinase activity as compared with those of wild-type v-Src and other Src mutant. When we treated these mutants Yes with $5 \mu \mathrm{M}$ NAM, C9AYes displayed a clear resistance to the treatment. In contrast, C6AYes showed suppression to some extent. Kinase activity of C6AYes after treatment was decreased to $\sim 70 \%$ of the activity of an untreated one. The difference between C6AYes and C9AYes to the treatment with NAM was more prominent at a higher concentration of NAM. When we treated these Yes kinases with $25 \mu \mathrm{M}$ NAM in Fig. 2B, C9AYes still displayed a clear resistance to the treatment. In contrast, kinase activity of C6AYes was almost completely lost by the treatment. It should be noted here, that, in contrast to Yes, single point mutation of cysteines in the CC-motif of Src was not sufficient, though double mutations of cysteines were required to confer the resistance on Src to the inactivation of the kinase activity by the treatment with NAM (5). Since Yes originally lacks the cysteine residue corresponding to Cys496 of Src, the point mutation we created in either C6A and C9AYes therefore yielded the lack of two cysteine residues which is observed in the CC-motif of Src. Notably, mutation at $\mathrm{C} 9$ of Yes resulted in a paired mutation similarly present in our previous Src mutant (5). These findings suggest that double mutation in paired cysteine residues yields a stronger resistance to NAM as compared to those in distant positions. In this respect, Cys506 in Yes plays an important role in the resistance to the inactivation of its kinase activity by the SH-alkylating agent.

C9A Lyn mutant shows resistance to kinase inactivation of Lyn by NAM. To explore the importance of cysteine residue corresponding to Cys498 of Src, we then constructed Lyn mutants, C7ALyn and C9ALyn, in which Cys468 and Cys479 corresponding to Cys487 and Cys498 of Src were substituted with alanines, respectively. Since Lyn lacks two of the four cysteine residues in the CC-motif of Src, Lyn displayed resistance to NAM treatment as the double mutant of Src showed (5). By treatment with $10 \mu \mathrm{M}$ NAM in Fig. 3A, wild-type Lyn showed partial resistance to kinase inactivation 
(A)

WT FAK C7A FAK C9A FAK
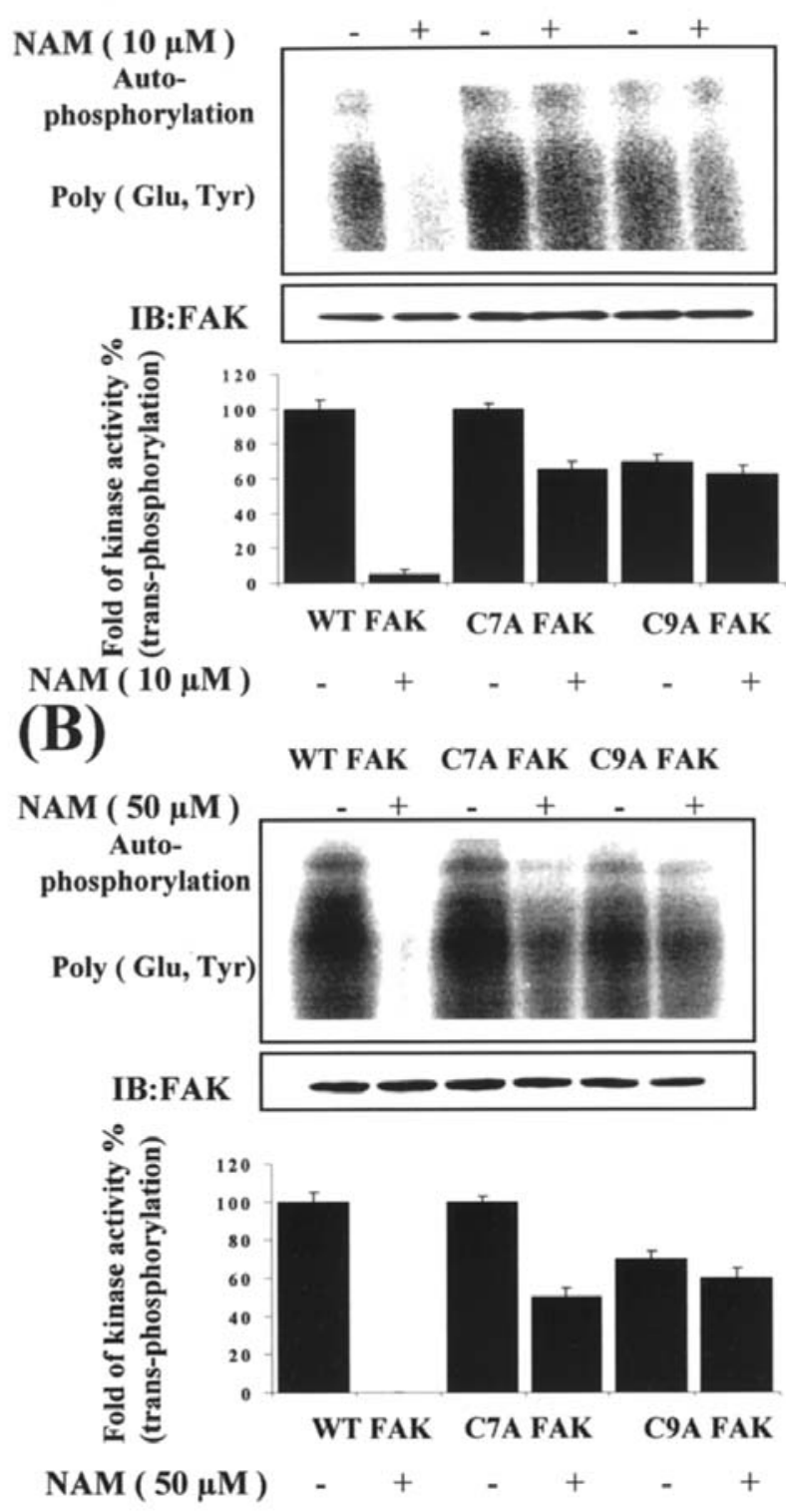

Figure 4. Effect of SH-alkylating agent, NAM, on the kinase activities of wild-type FAK and its mutants, C7AFAK and C9AFAK. (A) FAK and its mutants were immunoprecipitated from cell lysates by an anti-FAK antibody. An in vitro kinase assay of FAK in the presence or absence of $10 \mu \mathrm{M}$ of NAM was performed at $30^{\circ} \mathrm{C}$ for $2 \mathrm{~h}$ with poly (Glu,Tyr) as an exogenous substrate (upper panel). Immunoprecipitates were probed by anti-FAK for determination of an equal amount of loading (middle panel). Relative activities of the in vitro kinase assay were measured by transphosphorylation of poly (Glu,Tyr) and results were obtained from three independent assays (lower panel). (B) A higher dose of NAM $(50 \mu \mathrm{M})$ was used for the same experiment as above.

by NAM. In contrast, C9ALyn showed a substantial resistance to NAM. Although autophosphorylation of C9ALyn was suppressed by the NAM treatment, the activity to phosphorylate exogenous substrate, enolase, was retained well even after the treatment. The difference between wild-type Lyn and C9ALyn was more prominent at higher concentration of NAM. By treatment with $50 \mu \mathrm{M}$ NAM in Fig. 3B, wild-type Lyn was almost completely inactivated, whereas C9ALyn showed a clear resistance in the phosphorylation of exogenous substrate. NAM-treated C9ALyn retained $\sim 70 \%$ of trans- phosphorylation activity as compared with those of untreated C9ALyn whereas C7ALyn showed $\sim 50 \%$ resistance in the phosphorylation of exogenous substrate to $50 \mu \mathrm{M}$ NAM while wild-type Lyn was completely inactivated (Fig. 3C). These results are consistent with our previous observations (5) that double mutations in the pair of cysteines were required for resistance to the inactivation of the kinase activity against NAM treatment. As Lyn does not have two cysteines corresponding to Cys483 and Cys496 of Src, both C7A and C9ALyn lack two pairs of cysteine residues as compared with those of Src. The two mutants showed a clear resistance to the inactivation of the kinase activity of Lyn by NAM, though C9ALyn was more prominent in resistance than C7ALyn.

Effect of SH-alkylating agent, NAM, on the kinase activities of non-Src family kinase, FAK. As shown in Fig. 1A, FAK, which has a distinct structural difference from the Src-family kinase, also has the group 3-type $\mathrm{CC}$-motif in the $\mathrm{C}$-terminal side of the kinase domain. We then examined whether the modification of cysteine residues of the CC-motif in FAK by the SH-alkylating agent has a similar suppressive function on the kinase. FAK has two cysteine residues in its CC-motif, Cys647 and Cys658, which correspond to Cys487 and Cys498 of Src. Then we constructed two types of FAK mutants, C7AFAK and C9AFAK. In C7AFAK and C9AFAK, Cys647 and Cys658 of FAK were replaced with alanines by site-directed mutagenesis, respectively. The kinase activity of these mutants was compared with wild-type FAK with poly (Glu,Tyr) as an exogenous substrate. As shown in Fig. 4A, C9AFAK, although not C7AFAK, had reduced kinase activity as compared with that of wild-type FAK. Kinase activity of C9A mutant was $~ 80 \%$ of wild-type FAK. By treatment with $10 \mu \mathrm{M}$ NAM, wild-type FAK showed a clear suppression in kinase activity. In contrast, both C7AFAK and C9AFAK showed substantial resistance to the inactivation of the kinase activity of FAK by NAM, although resistance to kinase inactivation by NAM was more prominent in C9AFAK than in C7AFAK. To determine the kinase activity of FAK mutants upon a higher concentration of NAM, we treated wild-type FAK and mutants with $50 \mu \mathrm{M}$ NAM (Fig. 4B). Upon a higher concentration of NAM, C9A and C7AFAK mutant showed $\sim 80$ and $50 \%$ resistance, respectively in the phosphorylation of poly (Glu,Tyr) substrate while wild-type FAK was completely inactivated by NAM. These results are consistent with those of Lyn and cysteines corresponding to Cys487 and Cys498 of Src which are conserved well among the Src kinases as well as in FAK, indicating their critical role for the inhibition of kinase by the SH-alkylating agent.

\section{Discussion}

Since the protein tyrosine kinases play a critical role in a wide array of human carcinomas, development of their inhibitors is an important mission for the therapeutic progress of human malignancies. All kinases, however, share the same substrate, adenosine 5'-triphosphate (ATP) and molecular recognition determinants in their conserved nucleotide binding pocket so that no ATP analog that selectively inhibits a single kinase has been found (4). In this report, we showed that the CC-motif conserved only in the Src family kinases and FAK has an inhibitory function by the modification of cysteine residues 
with the SH-alkylating reagent. Our results strongly suggest that the CC-motif is a promising molecular target which can compensate the rational drug design of specific inhibitors for the kinases.

Based on the number of conserved cysteine residues in the CC-motif, tyrosine kinases can be classified into three groups. We found that $\mathrm{v}-\mathrm{Src}, \mathrm{c}-\mathrm{Src}$ and $\mathrm{c}-\mathrm{Yes}$ showed a clear sensitivity to the kinase activity by the treatment with $5 \mu \mathrm{M}$ NAM, whereas Lyn showed a substantial resistance to kinase inactivation from the treatment. These results confirm and extend our previous observations with Src that sensitivity of Src to the SH-alkylating reagents depended on the number of cysteines in the CC-motif and at least two point mutations in cysteine residues were required to confer resistance to kinase inactivation by the reagents (5). While Src and Yes have four or three conserved cysteine residues in the CC-motif, Lyn has only two of them.

Among the four cysteine residues conserved in the $\mathrm{CC}$ motif of Src, Cys498 appears to have an exceptionally important function for the kinase activity. Cys498 and Cys487 are most conserved cysteine residues among tyrosine kinases (Fig. 1A). We then found that a single point mutation at Cys498 of Src clearly caused a substantial reduction of the kinase activity and intracellular instability of the mutant protein $(5,6)$. In c-Yes, Lyn and FAK, single point mutations at cysteine residues corresponding to Cys498 of Src also rendered a reduction of their kinase activities, although the levels of reduction differ among these kinases. In contrast, single point mutations at other conserved cysteine residues including Cys487 did not cause a substantial reduction of the kinase activity. These results strongly suggest that, among the four cysteines conserved in the CC-motif, the cysteine residue that corresponding to Cys498 of Src, which is distant from the kinase cleft, has a conformationally important function for the kinase activity.

Since the Src-family kinase and FAK play an important role in human malignancies as well as acute and chronic inflammatory disorders, our study will be helpful in designing new therapeutic strategies in order to mitigate complications associated with malignancies and chronic inflammatory disorders.

\section{Acknowledgements}

We thank members of the Hamaguchi Laboratory for their technical assistance and helpful discussions. This study was supported by a Grant-in-Aid for COE research from the Ministry of Education, Science, Culture and Technology of Japan.

\section{References}

1. Martin GS: The hunting of the Src. Nat Rev Mol Cell Biol 2: 467-475, 2001.

2. Levitzki A: Protein tyrosine kinase inhibitors as novel therapeutic agents. Pharmacol Ther 82: 231-239, 1999.

3. Hunter T: Signaling-2000 and beyond. Cell 100: 113-127, 2000.

4. Ahn NG and Resing KA: Cell Biology: Lessons in rational drug design for protein kinases. Science 308: 1266-1267, 2005.

5. Oo ML, Senga T, Thant AA, Amin ARMR, Huang P, Mon NN and Hamaguchi M: Cysteine residues in the C-terminal lobe of Src: their role in the suppression of the Src kinase. Oncogene 22: 1411-1417, 2003

6. Senga T, Miyazaki K, Machida K, Iwata H, Matsuda S, Nakashima I and Hamaguchi M: Clustered cysteine residues in the kinase domain of v-Src: critical role for protein stability, cell transformation and sensitivity to herbimycin A. Oncogene 19: 273-279, 2000.

7. Yeatman TJ: A renaissance for Src. Nat Rev Cancer 4: 470-480, 2004.

8. Cooper JA and Howell B: The when and how of Src regulation. Cell 73: 1051-1054, 1993.

9. Sicheri F and Kuriyan J: Structures of Src-family tyrosine kinases. Curr Opin Struct Biol 7: 777-785, 1997.

10. Xu W and Harrison S: Three dimensional structure of the tyrosine kinase c-Src. Nature 385: 595-601, 1997.

11. Brown MT and Cooper JA: Regulation, substrates and functions of Src. Biochim Biophys Acta 1287: 121-149, 1996.

12. Lipsich LA, Lewis AJ and Brugge JS: Isolation of monoclonal antibodies that recognize the transforming proteins of avian sarcoma viruses. J Virol 48: 352-360, 1983.

13. Sukegawa J, Semba K, Yamanashi Y, Nishizawa M, Miyajima N, Yamamoto T and Toyoshima K: Characterization of cDNA clones for the human $c$-yes gene. Mol Cell Biol 7: 41-47, 1987.

14. Yamanashi Y, Fukushige S, Semba K, Sukegawa J, Miyajima N, Matsubara K, Yamamoto T and Toyoshima K: The yes-related cellular gene lyn encodes a possible tyrosine kinase similar to p56 ${ }^{\text {lck }}$. Mol Cell Biol 7: 237-243, 1987.

15. Hanks SK, Calalb MB, Harper MC and Patel SK: Focal adhesion protein-tyrosine kinase phosphorylated in response to cell attachment to fibronectin. Proc Natl Acad Sci USA 89: 8487-8491, 1992.

16. Mikaelian I and Sergeant A: A general and fast method to generate multiple site directed mutations. Nucleic Acids Res 20: 376, 1992.

17. Hamaguchi $\mathrm{M}$ and Hanafusa $\mathrm{H}$ : Association of p60src with Triton X-100-resistant cellular structure correlates with morphological transformation. Proc Natl Acad Sci USA 84: 2312-2316, 1987

18. Hamaguchi M, Matsuyoshi N, Ohnishi Y, Gotoh B, Takeichi M and Nagai Y: p60v-src causes tyrosine phosphorylation and inactivation of the $\mathrm{N}$-cadherin-catenin cell adhesion system. EMBO J 12: 307-314, 1993.

19. Hamaguchi M, Xiao H, Uehara Y, Ohnishi Y and Nagai Y: Herbimycin A inhibits the association of p60v-src with the cytoskeletal structure and with phosphatidylinositol 3' kinase. Oncogene 8: 559-564, 1993. 